\title{
Radiative Transfer Theory for Vacuum Fluctuations
}

\author{
E. G. Mishchenko ${ }^{1,2}$ and C. W. J. Beenakker ${ }^{1}$ \\ ${ }^{1}$ Instituut-Lorentz, Universiteit Leiden, P.O. Box 9506, 2300 RA Leiden, The Netherlands \\ ${ }^{2}$ L. D. Landau Institute for Theoretical Physics, Russian Academy of Sciences, Kosygin 2, Moscow 117334, Russia
}

(Received 6 July 1999)

\begin{abstract}
A semiclassical kinetic theory is presented for the fluctuating photon flux emitted by a disordered medium in thermal equilibrium. The kinetic equation is the optical analog of the Boltzmann-Langevin equation for electrons. Vacuum fluctuations of the electromagnetic field provide a new source of fluctuations in the photon flux, over and above the fluctuations due to scattering. The kinetic theory in the diffusion approximation is applied to the super-Poissonian noise due to photon bunching and to the excess noise due to beating of incident radiation with the vacuum fluctuations.
\end{abstract}

PACS numbers: 42.50.Ar, 05.40.-a, 42.68.Ay, 78.45.+h

The theory of radiative transfer was developed by Chandrasekhar [1] and Sobolev [2] to describe the scattering and absorption of electromagnetic radiation by interstellar matter. It has become widely used in the study of wave propagation in random media, with applications in medical imaging and seismic exploration [3]. The basic equation of radiative transfer theory is a kinetic equation of the Boltzmann type that is derived from the Maxwell equations by neglecting interference effects [4]. It is a reliable approximation whenever the scattering and absorption lengths are large compared to the wavelength, which applies to all but the most strongly disordered media.

Radiative transfer theory has so far been restricted to classical waves, excluding purely quantum mechanical effects of vacuum fluctuations. This limitation is felt strongly in connection with the recent activity on random lasers [5]. These are amplifying systems in which the feedback is provided by multiple scattering from disorder rather than by mirrors, so that radiative transfer theory is an appropriate level of description. However, while stimulated emission has been incorporated into this approach a long time ago by Letokhov [6], spontaneous emission has not. It is the purpose of our work to remove this limitation, by presenting an extension of the radiative transfer equation that includes vacuum fluctuations and the associated spontaneous emission of radiation.

Our inspiration came from the field of electronic conduction in disordered metals, where the notion of a fluctuating Boltzmann equation (or Boltzmann-Langevin equation) has been developed extensively [7-9], following the original proposal by Kadomtsev [10]. In that context the fluctuations originate from random scattering and they conserve the particle number. This same class of fluctuations exists also in the optical context considered here, but with a different correlator because of the difference between boson and fermion statistics. In addition, the photons have a new class of fluctuations, without particle conservation, originating from random absorption and emission events. Vacuum fluctuations are of the second class. We will extend the radiative transfer theory to include both classes of fluctuations. To demonstrate the validity of our "Boltzmann-Langevin equation for photons," we solve the problem of the excess noise from vacuum fluctuations in a waveguide geometry, for which an independent solution is known [11]. We then apply it to the unsolved problem of the thermal radiation from a spherical random medium.

The basic quantity of the kinetic theory is the fluctuating distribution function $f_{\mathbf{k}}(\mathbf{r}, t)$ of the number of photons per unit cell $(2 \pi)^{-3} d \mathbf{k} d \mathbf{r}$ in phase space. (For simplicity, we ignore the polarization dependence.) Conventional radiative transfer theory deals with the mean $\bar{f}_{\mathbf{k}}(\mathbf{r})$, which we assume to be time independent. It satisfies the Boltzmann equation

$$
\begin{aligned}
c \hat{\mathbf{k}} \cdot \frac{\partial \bar{f}_{\mathbf{k}}}{\partial \mathbf{r}}= & \sum_{\mathbf{k}^{\prime}}\left[J_{\mathbf{k} \mathbf{k}^{\prime}}(\bar{f})-J_{\mathbf{k}^{\prime} \mathbf{k}}(\bar{f})\right]+I_{\mathbf{k}}^{+}(\bar{f}) \\
& -I_{\mathbf{k}}^{-}(\bar{f}) .
\end{aligned}
$$

[For ease of notation, we write $\sum_{\mathbf{k}}$ instead of $(2 \pi)^{-3} \int d \mathbf{k}$, and $\delta_{\mathbf{k q}}$ instead of $(2 \pi)^{3} \delta(\mathbf{k}-\mathbf{q})$.] The left-hand side is the convection term (with $c$ the velocity of light in the medium and $\hat{\mathbf{k}}$ a unit vector in the direction of the wave number $\mathbf{k})$. The right-hand side contains gain and loss terms due to scattering, $J_{\mathbf{k k}^{\prime}}(\bar{f})=w_{\mathbf{k} \mathbf{k}^{\prime}} \bar{f}_{\mathbf{k}^{\prime}}\left(1+\bar{f}_{\mathbf{k}}\right)$, due to amplification, $I_{\mathbf{k}}^{+}(\bar{f})=w_{\mathbf{k}}^{+}\left(1+\bar{f}_{\mathbf{k}}\right)$, and due to absorption $I_{\mathbf{k}}^{-}(\bar{f})=w_{\mathbf{k}}^{-} f_{\mathbf{k}}$. The scattering rate $w_{\mathbf{k} \mathbf{k}^{\prime}}=$ $w_{\mathbf{k}^{\prime} \mathbf{k}}$ is elastic and symmetric. The absorption and amplification rates $w_{\mathbf{k}}^{ \pm}$are isotropic (dependent only on $k=$ $|\mathbf{k}|)$ and related to each other by the requirement that the Bose-Einstein function

$$
f_{\text {eq }}(\omega, T)=\left[\exp \left(\hbar \omega / k_{B} T\right)-1\right]^{-1}
$$

is the equilibrium solution of Eq. (1) (at frequency $\omega=$ $c k$ and temperature $T$ ). This requirement fixes the ratio $w_{\mathbf{k}}^{-} / w_{\mathbf{k}}^{+}=\exp \left(\hbar \omega / k_{B} T\right)$. The temperature $T$ is positive for an absorbing medium and negative for an amplifying medium such as a laser [12].

We now extend the radiative transfer equation (1) to include the fluctuations $\delta f=f-\bar{f}$. Following the line of argument that leads to the Boltzmann-Langevin equation 
for electrons [7-10], we propose the kinetic equation

$$
\begin{aligned}
c \hat{\mathbf{k}} \cdot \frac{\partial f_{\mathbf{k}}}{\partial \mathbf{r}}= & \sum_{\mathbf{k}^{\prime}}\left[J_{\mathbf{k} \mathbf{k}^{\prime}}(f)-J_{\mathbf{k}^{\prime} \mathbf{k}}(f)\right] \\
& +I_{\mathbf{k}}^{+}(f)-I_{\mathbf{k}}^{-}(f)+\mathcal{L}_{\mathbf{k}} .
\end{aligned}
$$

The argument is that the fluctuating $f$ is propagated, scattered, absorbed, and amplified in the same way as the mean $\bar{f}$, hence the same convection term and the same kernels $J_{\mathbf{k k}^{\prime}}, I_{\mathbf{k}}^{ \pm}$appear in Eqs. (1) and (3). In addition, Eq. (3) contains a stochastic source of photons,

$$
\mathcal{L}_{\mathbf{k}}=\sum_{\mathbf{k}^{\prime}}\left(\delta J_{\mathbf{k k}^{\prime}}-\delta J_{\mathbf{k}^{\prime} \mathbf{k}}\right)+\delta I_{\mathbf{k}}^{+}-\delta I_{\mathbf{k}}^{-},
$$

consisting of separate contributions from scattering, amplification, and absorption. This Langevin term has zero mean, $\overline{\mathcal{L}}_{\mathbf{k}}=0$, and a correlator that follows from the assumption that the elementary stochastic processes $\delta J_{\mathbf{k k}^{\prime}}, \quad \delta I_{\mathbf{k}}^{ \pm}$have independent Poisson distributions:

$$
\begin{aligned}
& \overline{\delta J_{\mathbf{k} \mathbf{k}^{\prime}}(\mathbf{r}, t) \delta J_{\mathbf{q} \mathbf{q}^{\prime}}\left(\mathbf{r}^{\prime}, t^{\prime}\right)}=\Delta \delta_{\mathbf{k q}} \delta_{\mathbf{k}^{\prime} \mathbf{q}^{\prime}} J_{\mathbf{k} \mathbf{k}^{\prime}}(\bar{f}), \\
& \overline{\delta I_{\mathbf{k}}^{ \pm}(\mathbf{r}, t) \delta I_{\mathbf{q}}^{ \pm}\left(\mathbf{r}^{\prime}, t^{\prime}\right)}=\Delta \delta_{\mathbf{k q}} I_{\mathbf{k}}^{ \pm}(\bar{f}), \\
& \overline{\delta J_{\mathbf{k} \mathbf{k}^{\prime}}(\mathbf{r}, t) \delta I_{\mathbf{q}}^{ \pm}\left(\mathbf{r}^{\prime}, t^{\prime}\right)}=0, \quad \overline{\delta I_{\mathbf{k}}^{+}(\mathbf{r}, t) \delta I_{\mathbf{q}}^{-}\left(\mathbf{r}^{\prime}, t^{\prime}\right)}=0,
\end{aligned}
$$

where we have abbreviated $\Delta=\delta\left(\mathbf{r}-\mathbf{r}^{\prime}\right) \delta\left(t-t^{\prime}\right)$. Substitution into Eq. (4) gives the correlator

$$
\overline{\mathcal{L}_{\mathbf{k}}(\mathbf{r}, t) \mathcal{L}_{\mathbf{q}}\left(\mathbf{r}^{\prime}, t^{\prime}\right)}=\Delta\left[\delta_{\mathbf{k q}} \sum_{\mathbf{k}^{\prime}}\left[J_{\mathbf{k} \mathbf{k}^{\prime}}(\bar{f})+J_{\mathbf{k}^{\prime} \mathbf{k}}(\bar{f})\right]-J_{\mathbf{k q}}(\bar{f})-J_{\mathbf{q} \mathbf{k}}(\bar{f})+\delta_{\mathbf{k q}}\left[I_{\mathbf{k}}^{+}(\bar{f})+I_{\mathbf{k}}^{-}(\bar{f})\right]\right] .
$$

Equations (3) and (6) constitute the Boltzmann-Langevin equation for photons.

To gain more insight into this kinetic equation we make the diffusion approximation valid if the mean free path is the shortest length scale in the system (but still large compared to the wavelength). The diffusion approximation consists in an expansion with respect to $\hat{\mathbf{k}}$ in spherical harmonics, keeping only the first two terms: $f_{\mathbf{k}}=$ $f_{0}+\hat{\mathbf{k}} \cdot \mathbf{f}_{1}, \mathcal{L}_{\mathbf{k}}=\mathcal{L}_{0}+\hat{\mathbf{k}} \cdot \mathcal{L}_{1}$, where $f_{0}, \mathbf{f}_{1}, \mathcal{L}_{0}$, and $\mathcal{L}_{1}$ do not depend on the direction $\hat{\mathbf{k}}$ of the wave vector, but on its magnitude $k=\omega / c$ only. The two terms $f_{0}$ and $\mathbf{f}_{1}$ determine, respectively, the photon number density $n=\rho f_{0}$ and flux density $\mathbf{j}=\frac{1}{3} c \rho \mathbf{f}_{1}$, where $\rho(\omega)=$ $4 \pi \omega^{2}(2 \pi c)^{-3}$ is the density of states. Integration of Eq. (3) gives two relations between $n$ and $\mathbf{j}$,

$$
\begin{gathered}
\mathbf{j}=-D \frac{\partial n}{\partial \mathbf{r}}+\frac{1}{3} l \rho \mathcal{L}_{1}, \\
\frac{\partial}{\partial \mathbf{r}} \cdot \mathbf{j}=D \xi_{a}^{-2}\left(\rho f_{\mathrm{eq}}-n\right)+\rho \mathcal{L}_{0},
\end{gathered}
$$

where the diffusion constant $D=\frac{1}{3} c^{2} \tau$ and mean free path $l=c \tau$ are determined by the transport scattering rate $\tau^{-1}=\sum_{\mathbf{k}^{\prime}} w_{\mathbf{k k}^{\prime}}\left(1-\hat{\mathbf{k}} \cdot \hat{\mathbf{k}}^{\prime}\right)$. The absorption length $\xi_{a}$ is defined by $D \xi_{a}^{-2}=w^{-}-w^{+}$. (An amplifying medium has an imaginary $\xi_{a}$ and a negative $f_{\text {eq. }}$.) In Eq. (7) we have neglected terms of order $\left(l / \xi_{a}\right)^{2}$, which are assumed to be $\ll 1$.

Both Eqs. (7) and (8) contain a fluctuating source term. These two terms $\mathcal{L}_{0}$ and $\mathcal{L}_{1}$ have zero mean and correlators that follow from Eq. (6),

$$
\overline{\mathcal{L}_{0}(\omega, \mathbf{r}, t) \mathcal{L}_{0}\left(\omega^{\prime}, \mathbf{r}^{\prime}, t^{\prime}\right)}=\Delta^{\prime} \frac{D}{\rho \xi_{a}^{2}}\left(2 f_{\text {eq }} \bar{f}_{0}+f_{\text {eq }}+\bar{f}_{0}\right),
$$

$$
\overline{\mathcal{L}_{1}(\omega, \mathbf{r}, t) \mathcal{L}_{1}\left(\omega^{\prime}, \mathbf{r}^{\prime}, t^{\prime}\right)}=\mathbb{1} \Delta^{\prime} \frac{6 c}{\rho l} \bar{f}_{0}\left(1+\bar{f}_{0}\right),
$$

$$
\overline{\mathcal{L}_{0}(\omega, \mathbf{r}, t) \mathcal{L}_{1}\left(\omega^{\prime}, \mathbf{r}^{\prime}, t^{\prime}\right)}=\Delta^{\prime} \frac{D}{\rho \xi_{a}^{2}}\left(2 f_{\mathrm{eq}} \bar{f}_{1}+\bar{f}_{1}\right),
$$

where we have abbreviated $\Delta^{\prime}=\delta\left(\omega-\omega^{\prime}\right) \delta(t-$ $\left.t^{\prime}\right) \delta\left(\mathbf{r}-\mathbf{r}^{\prime}\right)$. The correlator (9b) differs from the electronic case [13-15] by the factor $1+\bar{f}_{0}$ instead of $1-\bar{f}_{0}$. This is the expected difference between boson and fermion statistics. The correlators (9a) and (9c) have no electronic counterpart. They describe the statistics of the vacuum fluctuations.

To demonstrate how the kinetic theory presented above works in a specific situation we consider the propagation through an absorbing or amplifying disordered waveguide (length $L$ ). The incident radiation is isotropic. All transmitted radiation is absorbed by a photodetector (see Fig. 1). Because of the one dimensionality of the geometry we need to consider only the $x$ dependence of $\mathbf{j}$ and $n$ (we assume a unit cross-sectional area). The transmitted photon flux $I=\int_{0}^{\infty} d \omega j(\omega, L, t)$ fluctuates around its time-averaged value, $I(t)=\bar{I}+\delta I(t)$. The (zero-frequency) noise power $P=\int_{-\infty}^{\infty} d t \overline{\delta I(t) \delta I(0)}$ is the correlator of the fluctuating flux. We will compute $P$ by solving the differential equations (7) and (8) with boundary conditions $n(\omega, 0, t)=n_{\text {in }}(\omega, t), n(\omega, L, t)=0$, dictated by the incident radiation at one end of the waveguide and by the absorbing photodetector at the other end.

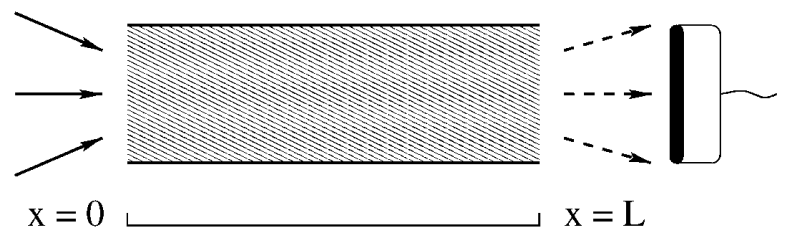

FIG. 1. Isotropic radiation (solid arrows) is incident on a waveguide containing an absorbing or amplifying random medium. The transmitted radiation (dashed arrows) is absorbed by a photodetector. 
Combining Eqs. (7) and (8) we find equations for the mean and the fluctuations of the photon number,

$$
\begin{gathered}
\frac{d^{2} \bar{n}}{d x^{2}}-\frac{\bar{n}}{\xi_{a}^{2}}=-\frac{\rho f_{\mathrm{eq}}}{\xi_{a}^{2}}, \\
\frac{d^{2} \delta n}{d x^{2}}-\frac{\delta n}{\xi_{a}^{2}}=\frac{\rho}{c} \frac{d \mathcal{L}_{1}}{d x}-\frac{\rho \mathcal{L}_{0}}{D} .
\end{gathered}
$$

The homogeneous differential equation has Green function

$$
G\left(x, x^{\prime}\right)=-\xi_{a} \frac{\sinh \left(x_{<} / \xi_{a}\right) \sinh \left(s-x_{>} / \xi_{a}\right)}{\sinh s},
$$

where we have defined $s=L / \xi_{a}$ and $x_{<}\left(x_{>}\right)$is the smallest (largest) of $x$ and $x^{\prime}$. (In the amplifying system $\xi_{a}$ is imaginary so the hyperbolic functions become trigonometric functions.) The inhomogeneous equations (10) and (11) have the solution

$$
\begin{gathered}
\bar{n}(\omega, x)=\frac{\rho f_{\mathrm{eq}}}{\sinh s}\left[\sinh s-\sinh \left(x / \xi_{a}\right)-\sinh \left(s-x / \xi_{a}\right)\right] \\
+\bar{n}_{\mathrm{in}}(\omega) \frac{\sinh \left(s-x / \xi_{a}\right)}{\sinh s}, \\
\delta n(\omega, x, t)=\rho \int_{0}^{L} d x^{\prime} G\left(x, x^{\prime}\right)\left(\frac{1}{c} \frac{d \mathcal{L}_{1}}{d x^{\prime}}-\frac{\mathcal{L}_{0}}{D}\right) \\
+\delta n_{\mathrm{in}}(\omega, t) \frac{\sinh \left(s-x / \xi_{a}\right)}{\sinh s} .
\end{gathered}
$$

The flux density at the photodetector follows from Eq. (7) at $x=L$,

$$
\begin{aligned}
\bar{j}(\omega, L)= & \frac{D \rho f_{\mathrm{eq}}}{\xi_{a}} \tanh (s / 2)+\frac{D \bar{n}_{\mathrm{in}}}{\xi_{a} \sinh s}, \\
\delta j(\omega, L, t)= & \frac{D \delta n_{\mathrm{in}}}{\xi_{a} \sinh s}+\frac{D \rho}{\sinh s} \int_{0}^{L} d x \\
& \times\left(\sinh \left(x / \xi_{a}\right) \frac{\mathcal{L}_{0}}{D}+\cosh \left(x / \xi_{a}\right) \frac{\mathcal{L}_{1}}{c \xi_{a}}\right) .
\end{aligned}
$$

[Notice that the extra term $\propto \mathcal{L}_{1}$ in Eq. (7) is canceled by the delta function in $\partial^{2} G / \partial x \partial x^{\prime}$.]

The time-averaged flux $\bar{I}=\bar{I}_{\text {in }}+\bar{I}_{\text {th }}$ is the sum of two contributions, the transmitted incident flux $\bar{I}_{\text {in }}=$ $\int_{0}^{\infty} d \omega D \bar{n}_{\text {in }} /\left(\xi_{a} \sinh s\right)$, and the thermal flux $\bar{I}_{\mathrm{th}}=$ $\int_{0}^{\infty} d \omega\left(D \rho f_{\mathrm{eq}} / \xi_{a}\right) \tanh (s / 2)$. The transmitted incident flux per frequency interval is a fraction $T=4 D /\left(c \xi_{a} \sinh s\right)$ of the incident flux density $j_{0}=$ $\frac{1}{4} c \bar{n}_{\text {in }}$. A fraction $R=1-4 D /\left(c \xi_{a} \tanh s\right)$ of the incident flux is reflected. The thermal flux per frequency interval is a fraction $1-T-R=\left(4 D / c \xi_{a}\right) \tanh (s / 2)$ of the blackbody flux density $j_{0}=\frac{1}{4} c \rho f_{\text {eq }}$. This is Kirchhoff's law of thermal radiation.

The noise power $P$ follows from the autocorrelators of $\mathcal{L}_{0}$ and $\mathcal{L}_{1}$ [given by Eq. (9), with $\bar{f}_{0}=\bar{n}_{0} / \rho$ from Eq. (13)]. The autocorrelator of $\delta n_{\text {in }}$ and the cross correlator of $\mathcal{L}_{0}$ and $\mathcal{L}_{1}$ contribute only to order $\left(l / \xi_{a}\right)^{2}$ and can therefore be neglected. The noise power $P=P_{\text {in }}+$ $P_{\mathrm{th}}+P_{\mathrm{ex}}$ is found to consist of three terms, given by

$$
\begin{gathered}
P_{\text {in }}=\bar{I}_{\text {in }}+\int_{0}^{\infty} d \omega \frac{D \bar{n}_{\text {in }}^{2}}{8 \rho \xi_{a}} \frac{2 s \cosh (2 s)+\sinh (2 s)-4 s}{\sinh ^{4} s}, \\
P_{\mathrm{th}}=\bar{I}_{\mathrm{th}}+\int_{0}^{\infty} d \omega \frac{D \rho f_{\mathrm{eq}}^{2}}{4 \xi_{a}} \frac{\sinh ^{2}(s / 2)}{\sinh ^{4} s}[8 s+4 s \cosh s-7 \sinh s-4 \sinh (2 s)+\sinh (3 s)], \\
P_{\mathrm{ex}}=\int_{0}^{\infty} d \omega \frac{D f_{\mathrm{eq}} \bar{n}_{\mathrm{in}}}{2 \xi_{a}} \frac{\sinh ^{2}(s / 2)}{\sinh ^{4} s}[-6 s-4 s \cosh s+4 \sinh s+3 \sinh (2 s)] .
\end{gathered}
$$

The two terms $P_{\text {in }}$ and $P_{\text {th }}$ describe separately the fluctuations in the transmitted incident flux and in the thermal flux. Both terms are greater than the Poisson noise (the mean photon flux $\bar{I}_{\text {th }}, \bar{I}_{\text {in }}$ ) as a consequence of photon bunching. The third term $P_{\mathrm{ex}}$ is the excess noise which in a quantum optical formulation originates from the beating of the incident radiation with vacuum fluctuations in the medium [16]. Here we find this excess noise from the semiclassical radiative transfer theory. The expressions for $P_{\text {th }}$ and $P_{\text {ex }}$ in Eq. (17) are the same as those that follow from the fully quantum optical treatment $[11,17]$. This is a crucial test of the validity of the semiclassical theory. The expression for $P_{\text {in }}$ agrees with the quantum optical theory for the case that the incident radiation originates from a thermal source [18]. The case of coherent incident radiation is beyond the reach of radiative transfer theory.

We envisage a variety of applications for the Boltzmann-Langevin equation for photons obtained in this paper. Although we have concentrated here on the waveguide geometry, in order to be able to compare with results in the literature, the calculation of the noise power in the diffusion approximation can be readily generalized to arbitrary geometry. As an example, we give the noise power of the thermal radiation emitted by a sphere (per unit surface area),

$$
\begin{aligned}
P_{\mathrm{th}}= & \bar{I}_{\mathrm{th}}+\int_{0}^{\infty} d \omega \frac{2 D \rho f_{\mathrm{eq}}^{2} s^{2}}{\xi_{a} \sinh ^{4} s} \int_{0}^{s} d z\left(\cosh z-\frac{\sinh z}{z}\right)^{2} \\
& \times \frac{\sinh ^{2} z}{z^{2}},
\end{aligned}
$$

where $s=R / \xi_{a}$ is the ratio of the radius $R$ of the sphere and the absorption length $\xi_{a}$. The mean thermal flux is given by $\bar{I}_{\text {th }}=\int_{0}^{\infty} d \omega D \rho f_{\text {eq }} \xi_{a}^{-1}(\operatorname{coth} s-1 / s)$. The result for $\bar{I}_{\text {th }}$ could have been obtained from the conventional radiative transfer theory using Kirchhoff's law, but the result for $P_{\text {th }}$ could not. 
A dimensionless measure of the magnitude of the photon flux fluctuations is the Mandel parameter [19], $Q=(P-\bar{I}) / \bar{I}$. In a photocount experiment, counting $n$ photons in a time $t$ with unit quantum efficiency, the Mandel parameter is obtained from the mean photocount $\bar{n}$ and the variance varn in the long-time limit: $Q=\lim _{t \rightarrow \infty}(\operatorname{var} n-\bar{n}) / \bar{n}$. We assume a frequency-resolved measurement, so that the integrals over frequency in Eqs. (17) and (18) can be omitted. The Mandel parameter for thermal radiation from a waveguide and a sphere is plotted in Fig. 2, as a function of $s\left(s=L / \xi_{a}\right.$ for the waveguide and $s=R / \xi_{a}$ for the sphere). Both the small- and large-s behavior of $Q$ is geometry independent: $Q=\frac{2}{15} s^{2} f_{\text {eq }}$ for $s \ll 1$ and $Q=\frac{1}{2} f_{\text {eq }}$ for $s \gg 1$. The Bose-Einstein function $f_{\mathrm{eq}}(\omega, T)$ is to be evaluated at the detection frequency $\omega$ and temperature $T$ of the medium. The plot in Fig. 2 is for $f_{\text {eq }}=10^{-3}$, typical for optical frequencies at $3000 \mathrm{~K}$.

Much larger Mandel parameters can be obtained in amplifying systems, such as a random laser. Since complete population inversion corresponds to $T \rightarrow 0^{-}$, one has $f_{\text {eq }}=-1$ in that case [12]. Equations (17) and (18) apply to amplified spontaneous emission below the laser threshold if one uses an imaginary $\xi_{a}$. The absolute value $\left|\xi_{a}\right|$ is the amplification length, and we denote $s=L /\left|\xi_{a}\right|$ for the waveguide geometry and $s=R /\left|\xi_{a}\right|$ for the sphere. The laser threshold occurs at $s=\pi$ in both geometries. We have included in Fig. 2 the Mandel parameter for these two amplifying systems for the case of complete population inversion. Again the result is geometry independent for small $s, Q=\frac{2}{15} s^{2}\left|f_{\text {eq }}\right|$ for $s \ll 1$. At the laser threshold $(s=\pi)$ the Mandel parameter diverges in the theory considered here. An important extension for future work is to include the nonlinearities that become of crucial

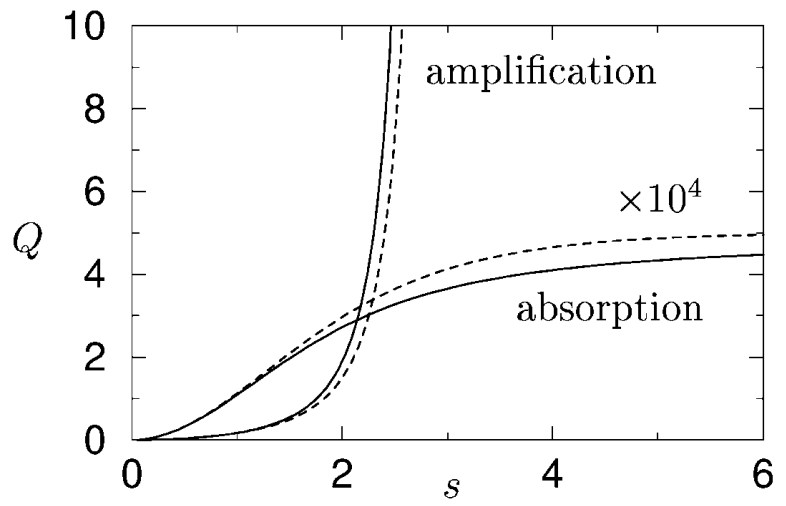

FIG. 2. Mandel parameter $Q=(P-\bar{I}) / \bar{I}$ for the thermal radiation from an absorbing medium and for the amplified spontaneous emission from a medium with a complete population inversion. The solid curves are for the sphere geometry [Eq. (18)]; the dashed curves are for the waveguide geometry [Eq. (17b)]. The parameter $s$ is the ratio of the radius of the sphere or of the length of the waveguide to the absorption or amplification length. The laser threshold in the amplifying case is at $s=\pi$. To show both cases in one figure, the $Q$ for the absorbing medium has been rescaled by a factor of $10^{4}$ (corresponding to $f_{\mathrm{eq}}=10^{-3}$ ). importance above the laser threshold. The simplicity of the radiative transfer theory developed here makes it a promising tool for the exploration of the nonlinear regime in a random laser.

Since radiative transfer theory was originally developed for applications in astrophysics, we imagine that the extension to fluctuations presented here could be useful in that context as well.

We acknowledge discussions with M. Patra. This work was supported by the Dutch Science Foundation NWO/FOM.

[1] S. Chandrasekhar, Radiative Transfer (Dover, New York, 1960).

[2] V. V. Sobolev, A Treatise on Radiative Transfer (Van Nostrand, Princeton, 1963).

[3] Diffuse Waves in Complex Media, edited by J.-P. Fouque, NATO ASI Ser. C531 (Kluwer, Dordrecht, 1999).

[4] M. C. W. van Rossum and T. M. Nieuwenhuizen, Rev. Mod. Phys. 71, 313 (1999).

[5] H. Cao, Y. G. Zhao, S. T. Ho, E. W. Seelig, Q. H. Wang, and R. P. H. Chang, Phys. Rev. Lett. 82, 2278 (1999). Earlier work on random lasers has been reviewed by D. Wiersma and A. Lagendijk, Phys. World 1, 33 (1997).

[6] V.S. Letokhov, Zh. Eksp. Teor. Fiz. 53, 1442 (1967) [Sov. Phys. JETP 26, 835 (1968)].

[7] Sh. M. Kogan and A. Ya. Shul'man, Zh. Eksp. Teor. Fiz. 56, 862 (1969) [Sov. Phys. JETP 29, 467 (1969)].

[8] S. V. Gantsevich, V.L. Gurevich, and R. Katilius, Riv. Nuovo Cimento 2(5), 1 (1979).

[9] Sh. Kogan, Electronic Noise and Fluctuations in Solids (Cambridge University Press, Cambridge, 1996).

[10] B. B. Kadomtsev, Zh. Eksp. Teor. Fiz. 32, 943 (1957) [Sov. Phys. JETP 5, 771 (1957)].

[11] M. Patra and C. W. J. Beenakker, Phys. Rev. A 60, 4059 (1999).

[12] The negative temperature of an amplifying medium is determined by the relative population of the upper and lower levels of the atomic transition, $N_{\text {lower }} / N_{\text {upper }}=$ $\exp \left(\hbar \Omega / k_{B} T\right)$, where $\Omega$ is the transition frequency. A complete population inversion corresponds to $T \rightarrow 0^{-}$.

[13] K.E. Nagaev, Phys. Lett. A 169, 103 (1992).

[14] M. J. M. de Jong and C. W. J. Beenakker, Phys. Rev. B 51, 16867 (1995).

[15] E. V. Sukhorukov and D. Loss, Phys. Rev. B 59, 13054 (1999).

[16] C. H. Henry and R. F. Kazarinov, Rev. Mod. Phys. 68, 801 (1996).

[17] C. W. J. Beenakker, in Diffuse Waves in Complex Media (Ref. [3]).

[18] According to the quantum optical theory of Ref. [11], the integral over frequency in the expression for $P_{\text {in }}$ contains an additional factor $g=\left\langle a^{\dagger} a^{\dagger} a a\right\rangle\left\langle a^{\dagger} a\right\rangle^{-2}-1$, where $a$ is the annihilation operator of the incident radiation. Equation (17) corresponds to thermal radiation, when $g=1$, while $g=0$ for coherent radiation. The noise terms $P_{\text {th }}$ and $P_{\mathrm{ex}}$ are independent of $g$.

[19] L. Mandel and E. Wolf, Optical Coherence and Quantum Optics (Cambridge University Press, New York, 1995). 